\title{
FFA-CONTEXT AWARE ENERGY EFFICIENT ROUTING USING FAST REACTIVE AND ADAPTIVE ALGORITHM
}

\author{
P. Kavitha Rani and E. Kannan \\ Department of CSE, Vel Tech University, Chennai, India
}

Received 2013-09-19; Revised 2013-11-27; Accepted 2013-12-26

\begin{abstract}
A vital issue of routing is how to efficiently strengthen energy consumption of the whole network and to avoid the energy-hole which leads to node failure or node death; this scenario makes the network to work improperly and leads to network death, Here we proves our hypothesis with linearity between routing and linear principles. Our proposed work effectively utilizes the adaption of Optimized particle swarm algorithm (Fast Reactive and fast adaptive algorithm) in order to solve the routing problem in wireless sensor networks to avoid the energy hole. Our approach effectively defines the node leader based on energy level and path optimization for node traversing using particle swarm which effectively utilizes the solution for problem in linearity into routing problem. Our algorithm FFA redefines the particle swarm rules which are reliable in routing strategies and adapts to the working principle. The optimization in our algorithm is to balance energy level and to withstand for its context. This study proves the accuracy using our algorithm respectively by theoretical survey and analysis with simulated results.
\end{abstract}

Keywords: WSN, Wireless Sensor Network, Swarm Intelligence, FFA, BCO, ACO

\section{INTRODUCTION}

In traditional wireless communication model ranges very large scale in energy consumption and high complexity to route the packets (Rong et al., 2005; Visu et al., 2012; Sedighizadeh and Masehian, 2009; Sombuntham and Kachitvichayanukul, 2010; Ruyan et al., 2010; Guo and Jia, 2012). In recent development in wireless communication which yields the sensor node to develop for multi-function (Arif and Rani, 2012) largely in sense of mobility patterns, random mobility, Probabilistic mobility, Controlled mobility, Predictable mobility (Alfawaer et al., 2007).

Because of rapid development in WSN sensor node elements which is coupled with sensor, communication devices, data processing (pre + post) and communication channel are in forward range. Wireless sensor network differs from other wireless network such ad hoc and infrastructure based which enables wide area of research in the sensor networks (Visu et al., 2012;
Sedighizadeh and Masehian, 2009; Sombuntham and Kachitvichayanukul, 2010; Ruyan et al., 2010). Routing and optimality in energy consuming is one of the greatest challenges in the wireless sensor network.

\subsection{Source Oriented Approach in WSN}

Whenever an event is identified in WSN, the structure of the source node is announced by itself to all the sink nodes which are connected particularly to it base (Rong et al., 2005). This makes the sink node to propagate awareness for whole event and to utilize the resources, querying the availability, node transmission, mobility ratio, packet base aspect. This leads to free mobility of roaming sink base with the routing grid or routing information table (Shirkande and Vatti, 2013; Yong-Chang and Gang, 2008).

\subsection{Sink Oriented Approach in WSN}

Sink periodically disseminates its location information to the network. This enables sensors to

\section{Corresponding Author: P. Kavitha Rani, Department of CSE, Vel Tech University, Chennai, India}


direct their sensed information towards sink (Rong et al., 2005; Ruyan et al., 2010; Yong-Chang and Gang, 2008). Sink oriented approaches require periodic location updates to keep sources aware about its information (Yong-Chang and Gang, 2008).

Many new algorithms have been proposed for the routing problem in WSNs (Barabasi and Albert, 1999; Karp and Kung, 2000; Broch et al., 1999; Park and Corson, 1997; Baghyalakshmi et al., 2010; Kennedy and Eberhart, 1995; Shen et al., 2009; Kennedy et al., 2001; Osama et al., 2005). These routing contrivances have taken into consideration the intrinsic topographies of WSNs along with the application and architecture rations. The task of finding and maintaining routes in WSNs is nontrivial since energy restrictions (Guo and Jia, 2012; Shirkande and Vatti, 2013) and sudden changes in node status (e.g., failure) cause frequent and unpredictable topological changes. To minimize latency and energy consumption, routing techniques proposed in this literature employ some well-known routing tactics e.g., data aggregation and clustering (Rao et al., 2003; Baghyalakshmi et al., 2010). Ant Colony Optimization (ACO) algorithm in swarm intelligence is one of the most important heuristics based optimization method which was successfully applied in various complex problems.

\subsection{Full Description of WSN Routing Algorithm Based on Fast Reactive and Fast Adaptive Approach}

The algorithm selects routing through distributing flooding and gradient throughout the whole network. Our approach only needs the local information about the nodes, which emits the Particle Swarm characteristics (Guo and Jia, 2012) of decentralized control and dynamic morphing topologies. Parallelly it supports multipath routing which enhances the operability and very less energy consumption during packet transmission (Visu et al., 2012).

\subsection{FFA Estimation-Basic Approach}

Step 1: Create a population of agent called particle distributed over the range called $\mathrm{X}$

Step 2: Assign an objective function and evaluate the position of each particle

Step 3: Check for position: If the position of the object is best than the previous one update it

Step 4: Find out the best particle and evaluate it with the previous best one
Step 5: Update the velocity of the particle based on the below stated formulae Equation (1):

$$
\mathrm{V}_{\mathrm{i}}^{\mathrm{t}+1}=\mathrm{V}_{\mathrm{i}}^{\mathrm{t}}+\varnothing 1 \mathrm{U}_{1}^{\mathrm{t}}\left(\mathrm{pb} \mathrm{b}_{\mathrm{i}}^{\mathrm{t}}+\mathrm{x}_{\mathrm{i}}^{\mathrm{t}}\right)+\varnothing 2 \mathrm{U}_{2}^{\mathrm{t}}\left(\mathrm{gb_{ \textrm {i } } ^ { \mathrm { t } }}+\mathrm{x}_{\mathrm{i}}^{\mathrm{t}}\right)
$$

Step 6: Switch the particle to its new position based on the function stated below Equation (2):

$$
\mathrm{X}_{\mathrm{i}}^{\mathrm{t}+1}=\mathrm{X}_{\mathrm{i}}^{\mathrm{t}}+\mathrm{V}_{\mathrm{i}}^{\mathrm{t}+1}
$$

Step 7: Repeat the step 2 until it satisfies the basic stopping criteria:

$$
\mathrm{X}(1) *(2) * \ldots *
$$

Figure 1 states the inertia function of the particle swarm nodes. Based on the inertia weight parameter each node is investigated. Inertia weight can be changed so that each node in WSN (Kennedy et al., 2001; Osama et al., 2005; Murthy and Manoj, 2004; Lee and Knignt, 2005; Alfawaer et al., 2007) can be estimated to find adjacent nodes and partial adjacent nodes. According to She and Eberhart they stated the criterion function as follows:

$$
\mathrm{V}_{\mathrm{i}}^{\mathrm{t}+1}=\mathrm{WV}_{\mathrm{i}}^{\mathrm{t}}+\varnothing 1 \mathrm{U}_{1}^{\mathrm{t}}\left(\mathrm{pb} \mathrm{b}_{\mathrm{i}}^{\mathrm{t}}+\mathrm{x}_{\mathrm{i}}^{\mathrm{t}}\right)+\varnothing 2 \mathrm{U}_{2}^{\mathrm{t}}\left(\mathrm{gb_{ \textrm {i } } ^ { \mathrm { t } }}+\mathrm{x}_{\mathrm{i}}^{\mathrm{t}}\right)
$$

where, $\mathrm{W}$ denotes the weight of the nodes.

\subsection{Local Field}

In traditional old routing in wireless sensor network the node energy, computing capability, node optimization (Kennedy and Eberhart, 1995; Shen et al., 2009; Osama et al., 2005) and storage capacity are very limited due to its old methodology which focuses on detailed view on storing the data's in routing table (Satyanarayanan, 2007) in the whole network and updating the data between each interval of time leads to more complexity and failure of nodes. To overcome these issues in our approach each nodes hold on the information which are necessary about the node on the local field (Shirkande and Vatti, 2013; Yong-Chang and Gang, 2008; Visu and Kannan, 2013; Okdem and Karaboga, 2009; Arif and Rani, 2012). Information such as node id, source transceiver's id, base station, host parameters. Fast adaptive approach adapts to each situation and concentrates on link establishment and residual energy parameter in energy context. 


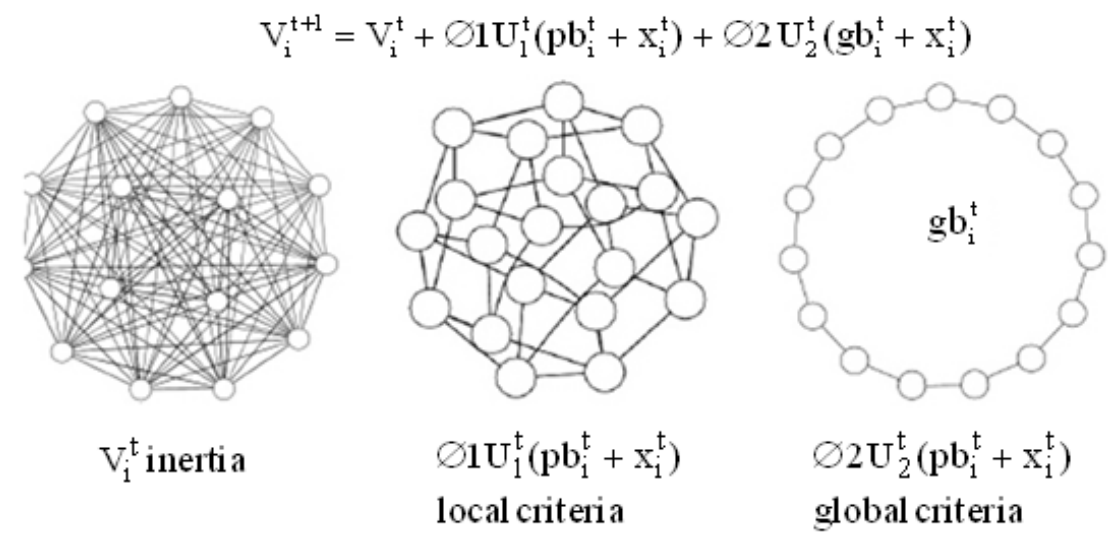

Fig. 1. Basic approach of Particle Swarm Optimization (PSO)

\subsection{FFA as Multipath Routing}

In our proposed approach each node records the activity between every node. The node calculates the likelihood values that each node is concentrated on particular node then it is evaluated over all the nodes. The node with positive and liable values stands and selects the nodes for the next jump. Hence it supports the multipath routing. Tracking adjacent nodes is denoted as follows:

$$
\mathrm{V}_{\mathrm{i}}^{\mathrm{t}+1}=\mathrm{x}\left[\mathrm{V}_{\mathrm{i}}^{\mathrm{t}}+\sum_{\rho \mathrm{k} \in \mathbb{\aleph} \mathrm{i}} \varphi_{\mathrm{k}} \mathrm{U}_{\mathrm{k}}^{\mathrm{t}}\left(p b_{\mathrm{k}}^{\mathrm{t}}+\mathrm{x}_{\mathrm{k}}^{\mathrm{t}}\right)\right]
$$

where, $\mathrm{V}_{\mathrm{i}}^{\mathrm{t}}$ denotes the velocity grid of the node.

Node-Head selection:

$$
V_{i}^{t}=A v^{t}+b 1 U_{1}^{t}\left(p b^{t}-x^{t}\right)+b 2 U_{2}^{t}\left(g b^{t}-x^{t}\right)
$$

Update the particle velocity rule in addition to the node element, consider the below:

$$
\begin{aligned}
& \mathrm{E}\left[\mathrm{U}_{*}^{\mathrm{t}}(0,1)\right]=\frac{1}{2} \\
& \mathrm{~B}=\frac{\mathrm{b} 1+\mathrm{b} 2}{2}
\end{aligned}
$$

To prove the routing linearity, considering the states of $\mathrm{pb}^{\mathrm{t}+1}$ and $\mathrm{gb}^{\mathrm{t}+1}$ :

$$
\begin{aligned}
& \mathrm{pb}^{\mathrm{t}+1}=\mathrm{pb}^{\mathrm{t}}+1, \mathrm{gb}^{\mathrm{t}+1}=\mathrm{gb}^{\mathrm{t}} \\
& \gamma=\frac{\mathrm{b} 1}{\mathrm{~b} 1+\mathrm{b} 2} \mathrm{pb}^{\mathrm{t}}+\frac{\mathrm{b} 1}{\mathrm{~b} 1+\mathrm{b} 2} \mathrm{gb}^{\mathrm{t}}
\end{aligned}
$$

Then the solution in terms of linear proposition:

$$
\mathrm{V}^{\mathrm{t}+1}=A v^{\mathrm{t}}+\mathrm{b}\left(\gamma-\mathrm{x}^{\mathrm{t}}\right)
$$

Hence $\mathrm{V}^{\mathrm{t}+1}$ is the velocity rule dominator which determines the node velocity. $\gamma$ Parameter determines the linearity of single dimension particle swarm value.

\subsection{Result Analysis}

Figure 2 shows the path optimization where the evaluation is based on each node and its ratio. Each fitness value is based on the estimation of the rows and its interlaced columns.

Experimentally, each iteration is carried on in order to achieve estimation of path parameters which is denoted in Fig. 3. Each energy is estimated on Numener value and its estimation with the accuracy rate of 5750 for every $\mathrm{k}(\mathrm{k}$ in terms of 1000) at each node.

Figure 5 Denotes the simulation of optimal route using FFA. Figure 4 denotes the cost evaluation matrix values for FFA algorithm. Results shows that our proposed algorithm has better efficiency in routing optimal path when compared to other existing algorithm. We achieved the accuracy and results has been justified in Fig. 4 and 5 (cost evaluation matrix and in routing simulation). 
P. Kavitha Rani and E. Kannan / American Journal of Applied Sciences 11 (2): 301-307, 2014

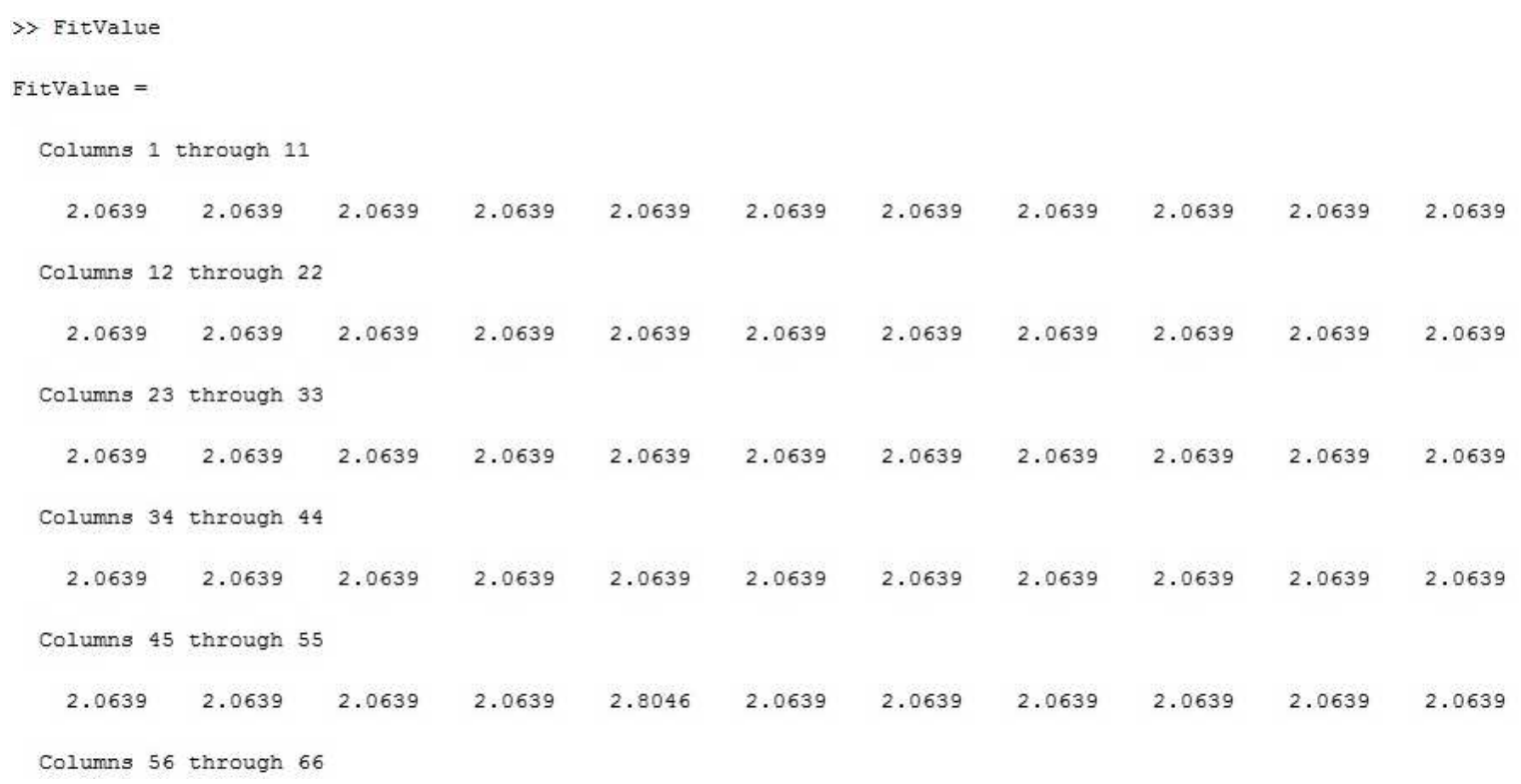

Fig. 2. Path optimization-Fitvalue for FFA Algorithm

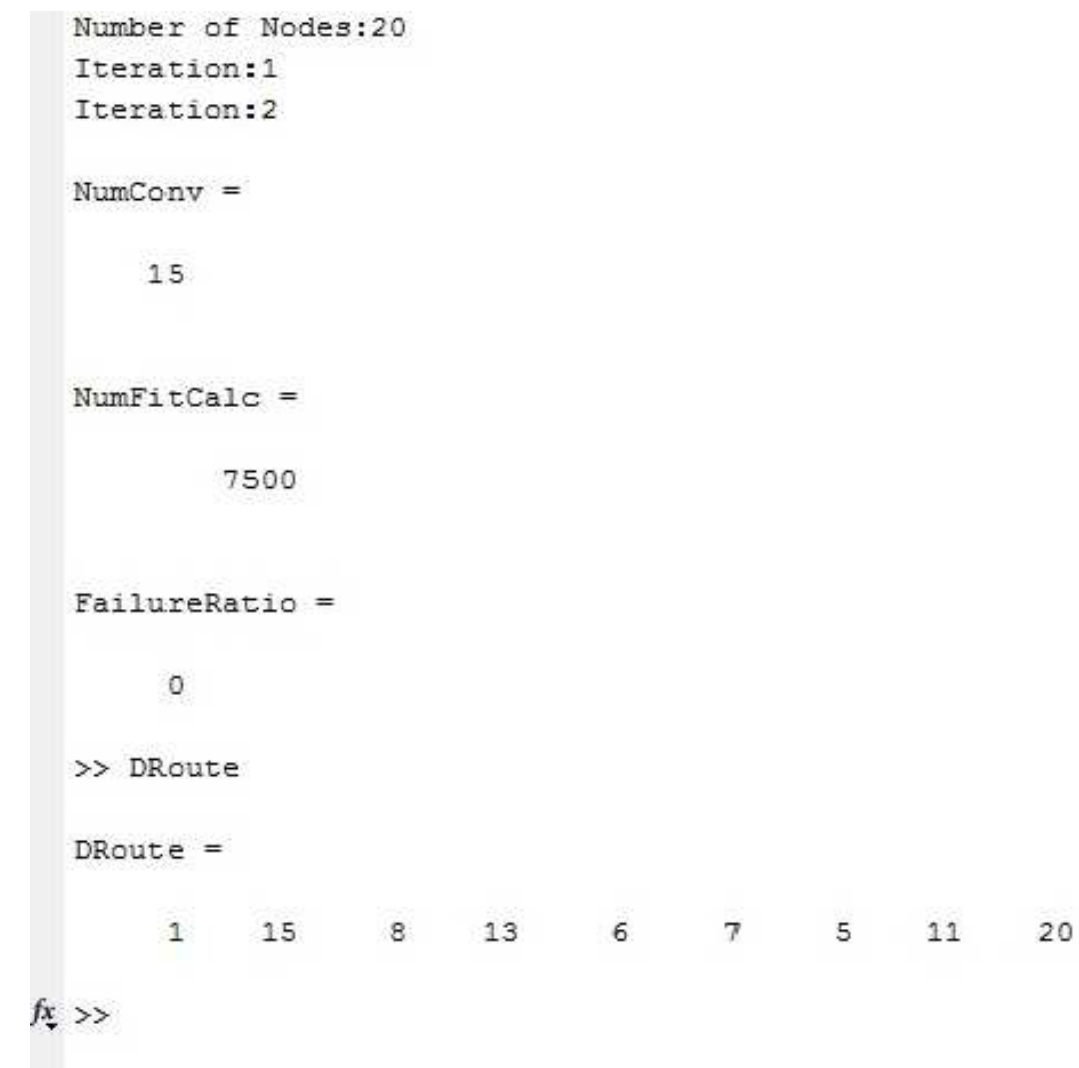

Fig. 3. Implementation results of FFA Route Algorithm 


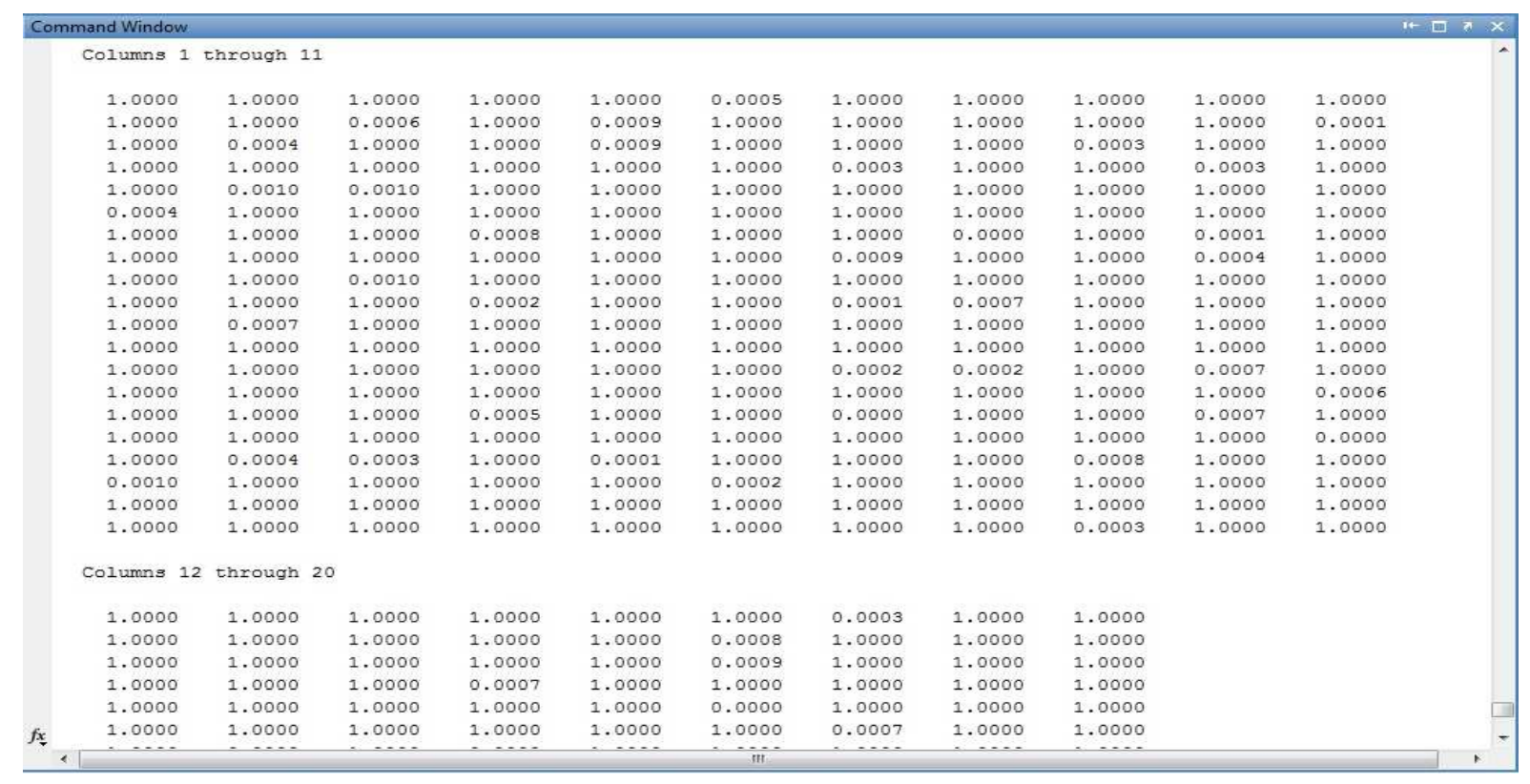

Fig. 4. Implementation results of cost evaluation matrix-FFA route algorithm

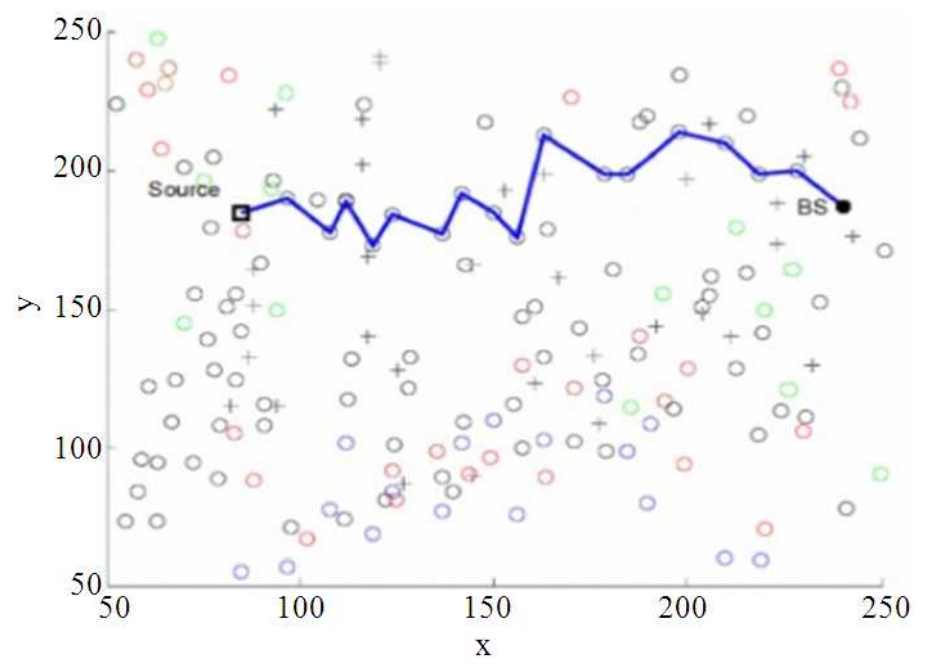

Fig. 5. Simulation result-FFA algorithm

\subsection{Comparison and Test Cases}

Basically Experimental setup is tested with 20 nodes and achieved accuracy for each in finding optimal path using FFA. Our acquired results has an exponential cut-off of about high accuracy of 11.5 optimality in terms of each iteration for 20 hops Failure estimation is based on each packet miss ratio, at every iteration failure hit is estimated, for every success failure hit value will be ' 0 '. The optimal prediction of results is achieved and the evaluation parameter is estimated based on real time packets, which the route was clearly defined in Fig. 3 as FFA Route. FFA has wide range of possibility to route the packets in mobile ad hoc networks, since each trackers seeds to provide the large number of packets in actual node (current state) real time example used in (mobile torrents). FFA uses multipath routing since the packets 
routed is within the node temp identity even if the node failure occurs, this leads to route the efficient packets (denoted clearly in the section multipath routing.

\section{CONCLUSION}

In this study we have discussed FFA based model in Wireless sensor network. Energy efficiency is one of the major criteria for deciding the efficacy of the routing protocol. Simulation results shows that the proposed routing methodologies have better optimality than the previous one. Here node header is clearly defined and optimized path is predicted with high accuracy in traversing lane. Figure denotes the accurate result of the proposed algorithm. Finally obtained FFA based routing method is the trade off between optimal measure and energy saving effect which can contribute to improve the robustness of the routing measure.

\subsection{Future Enhancement}

The main challenge in FFA algorithm is the algorithm route only for the predefined swarm nodes with average fit value, In future we like to combine routing algorithm in order to achieve better results for energy consumption in WSN; each swarm node is combined with hybrid routing in each extension.

\section{REFERENCES}

Alfawaer, Z.M., G.W. Hua, M.Y. Abdullah and I.D. Mamady, 2007. Power minimization algorithm in wireless ad hoc networks based on PSO. J. Applied Sci., $\quad$ 7: 2523-2526. DOI: 10.3923/jas.2007.2523.2526

Arif, M. and T. Rani, 2012. ACO based routing for MANETS. Singhania University.

Baghyalakshmi, D., J. Ebenezer and S.A.V. Satyamurty, 2010. Low latency and energy efficient routing protocols for wireless sensor networks. Proceedings of the International Conference on Wireless Communication and Sensor Computing, Jan. 2-4, IEEE Xplore Press, Chennai, pp: 1-6. DOI: 10.1109/ICWCSC.2010.5415892

Barabasi, A.L. and R. Albert, 1999. Emergence of scaling in random networks. Science, 286: 509-512. DOI: $10.1126 /$ science. 286.5439.509

Broch, J., D.B. Johnson and D.A. Maltz, 1999. The dynamic source routing protocol for mobile ad hoc networks. Internet-Draft Version 02, IETF.
Guo, D. and C. Jia, 2012. A WSN routing algorithm using overpayment on complex network. Proceedings of the 5th International Conference on Intelligent Computation Technology and Automation, Jan. 12-14, IEEE Xplore Press, Zhangjiajie, Hunan, pp: 53-56. DOI: 10.1109/ICICTA.2012.20

Kennedy, J. and R. Eberhart, 1995. Particle swarm optimization. Proceedings of the IEEE International Conference on Neural Networks, Nov. 27-Dec. 01, IEEE Xplore Press, Perth, WA., pp: 1942-1948. DOI: $10.1109 /$ ICNN.1995.488968

Kennedy, J.F., J. Kennedy and R.C. Eberhart, 2001. Swarm Intelligence. 1st Edn., Morgan Kaufmann, San Francisco, ISBN-10: 1558605959, pp: 512.

Lee, S. and D. Knignt, 2005. Realization of the nextgeneration network. IEEE Commun. Mag., 43: 3441. DOI: 10.1109/MCOM.2005.1522122

Murthy, C.S.R. and B.S. Manoj, 2004. Ad Hoc Wireless Networks: Architectures and Protocols. 1st Edn., Pearson Education India, ISBN-10: 8131759059.

Okdem, S. and D. Karaboga, 2009. Routing in wireless sensor networks using an Ant Colony Optimization (ACO) router chip. Sensors, 9: 909-921. DOI: 10.3390/s90200909

Osama, H.H., N.S. Tarek and J.L. Myung, 2005. Probability routing algorithm for mobile ad hoc networks' resources management. IEEE J. Selected Areas Commun., 23: 2248-2259. DOI: 10.1109/JSAC.2005.857205

Park, D.V. and M.S. Corson, 1997. A highly adaptive distributed routing algorithm for mobile wireless networks. Proceedings of the IEEE 16th Annual Joint Conference of the IEEE Computer and Communications Societies, Driving the Information Revolution, Apr. 7-12, IEEE Xplore Press, Kobe, pp: 1405-1413. DOI: 10.1109/INFCOM.1997.631180

Rao, A., S. Ratnasamy, C. Papadimitriou, S. Shenker and I. Stoica, 2003. Geographic routing without location information. Proceedings of the 9th Annual International Conference on Mobile Computing and Networking, Sep. 14-19, ACM Press, New York, USA., pp: 96-108. DOI: 10.1145/938985.938996

Rong, H., H. Zhigang and M. Hao, 2005. A reliable routing algorithm based on fuzzy applicability of $\mathrm{F}$ sets in MANET. Proceedings of the 11th Pacific Rim International Symposium on Dependable Computing, Dec. 12-14, IEEE Xplore Press. DOI: 10.1109/PRDC.2005.13 
Ruyan, Z., C. Ming, F. Guofu, L. Huifang and H. Shijun, 2010. Genetic clustering route algorithm in WSN. Proceedings of the 6th International Conference on Natural Computation, Aug. 10-12, IEEE Xplore Press, Yantai, Shandong, pp: 4023-4026. DOI: 10.1109/ICNC.2010.5584826

Sedighizadeh, D. and E. Masehian, 2009. Particle swarm optimization methods, taxonomy and application. Int. J. Comput. Theory Eng., 5: 486-502. DOI: 10.7763/IJCTE.2009.V1.80

Sombuntham, P. and V. Kachitvichayanukul, 2010. A particle swarm optimization algorithms for multidepot vehicle routing problem with pickup and delivery requests. Proceedings of the International Multi Conference of Engineers and Computer Science, Mar. 17-19, Hong Kong.

Shirkande, S.D. and R.A Vatti, 2013. ACO based routing algorithms for Ad-hoc Network (WSN, MANETs): A survey. Proceedings of the International Conference on Communication Systems and Network Technologies, Apr. 6-8, IEEE Xplore Press, Gwalior, pp: 230-235. DOI: 10.1109/CSNT.2013.56

Satyanarayanan, D., 2007. Greedy local Delaunay triangulation routing for wireless ad hoc networks. Proceedings of the International Conference on Signal Processing, Communications and Networking, Feb. 22-24, IEEE Xplore Press, Chennai, pp: 49-53. DOI: 10.1109/ICSCN.2007.350694
Shen, H., Y. Zhu, T. Liu and L. Jin, 2009. Particle swarm optimization in solving vehicle routing problem. Proceedings of the 2nd International Conference on Intelligent Computation Technology and Automation, Oct. 10-11, IEEE Xplore Press, Changsha, Hunan, pp: 287-291. DOI: 10.1109/ICICTA.2009.77

Visu, P., S. Koteeswaran and J. Janet, 2012. Artificial bee colony based energy aware and energy efficient routing protocol. J. Comput. Sci., 8: 227-231. DOI: 10.3844/jcssp.2012.227.231

Visu, P. and E. Kannan, 2013. Combined swarm intelligence routing protocol for MANET. Inform. Technol. J., 12: 791-797. DOI: 10.3923/itj.2013.791.797

Yong-Chang, Y.U. and W.E.I. Gang, 2008. Load balance data gathering algorithm in wireless sensor networks. J. Commun. Technol., 41: 92-95. 